\title{
THE CHANGE OF PRINTOUTS' QUALITY DEPENDING ON PRESSURE OF A BLANKET CYLINDER AGAINST AN IMPRESSION CYLINDER AND A PLATE CYLINDER IN OFFSET MACHINE
}

\author{
Agnieszka JURKIEWICZ; Yuriy PYR'YEV* \\ ${ }^{*}$ Faculty of Production Engineering, Institute of Mechanics and Printing, \\ Warsaw University of Technology, ul. Konwiktorska 2, 00-217 Warsaw, Poland \\ agnieszka.jurkiewicz@wp.eu, y.pyryev@wip.pw.edu.pl
}

\begin{abstract}
According to lots of books, the pressure in offset printing affects the optical density and the quality of printouts. One of the quality parameters is a tone value increase. An advantage of our research method is obtaining printing effects for different pressures on one printout, thus meaning for identical printing conditions. We obtained the same printing conditions through using different amounts of underlay sheets fixed to the blanket cylinder, under a blanket. The pressure was increased from optimal settings - in accordance with the machine manufacturer's recommendation. The test printouts were printed using Adast Dominant 515, on a coated and an uncoated paper. The optical density value was measured on the tone value scale from $10 \%$ to $100 \%$ stepping regularly by $10 \%$. For this scale the tone value increase was computed. The research shows that for both types of paper the optical density and the tone value increase changes not very much above the optimal pressure recommended by the machine manufacturer. A difference in the optical density and in the tone value increase is bigger for coated paper than for uncoated paper. Changes in these two parameters are negligible in places where used form 0 to 4 underlay sheets and are quite significant in the place where 5 underlay sheets were.
\end{abstract}

Key words: Optical Density, Tone Value Increase, Contact Between Cylinders

\section{INTRODUCTION}

A mathematical model of the contact problem between two rollers was described in the article (Jurkiewicz and Pyr'yev, 2011). Next, within further research the contact problem was examined in terms of the quality of printouts. The research results were described in this article. Theoretical model describes the relationshipof the compression and width of contact area between metal and rubber rollers. The compression of rollers causes increase of contact area. It is possible to evaluate the pressure between the cylinders. In the experimental research there is also a contact between rubber and metal surfaces of the cylinders. The research results demonstrate the effect of the cylinders compression on the quality of printouts.

A printing unit in an offset printing machine is built mainly of cylinders and rollers. There are three cylinders: a plate cylinder, a blanket cylinder and an impression cylinder (Fig. 1). In the offset printing machine there are smaller rollers (in the unking unit and in tha dampening unit) with closed circuit and bigger cylinders with a canal along the cylinder's axis. The canal comprises elements which fix a plate or a blanket or, alternatively, grippers which clip paper during printing.

During printing a dampening solution is conveyed by the dampening rollers from an ink fountain to the non-printing dots on the plate. Similarly, ink is conveyed from the ink fountain, triturated and then conveyed using ink rollers to the printing dots on the plate fixed to the plate cylinder. Next, the inked image is transferred from the plate cylinder to the blanket cylinder, and then from the blanket cylinder onto the paper clamped by the impression cylinder to the blanket cylinder.

The blanket cylinder has the blanket and the underlay sheets. There are two types of blankets: compressible and incompressi- ble. The blankets are made of a rubber top coating and fabric layers connected each to other with caoutchouc glue. The compressible blanket has an additional compressible sponge-like layer with air microbubbles.

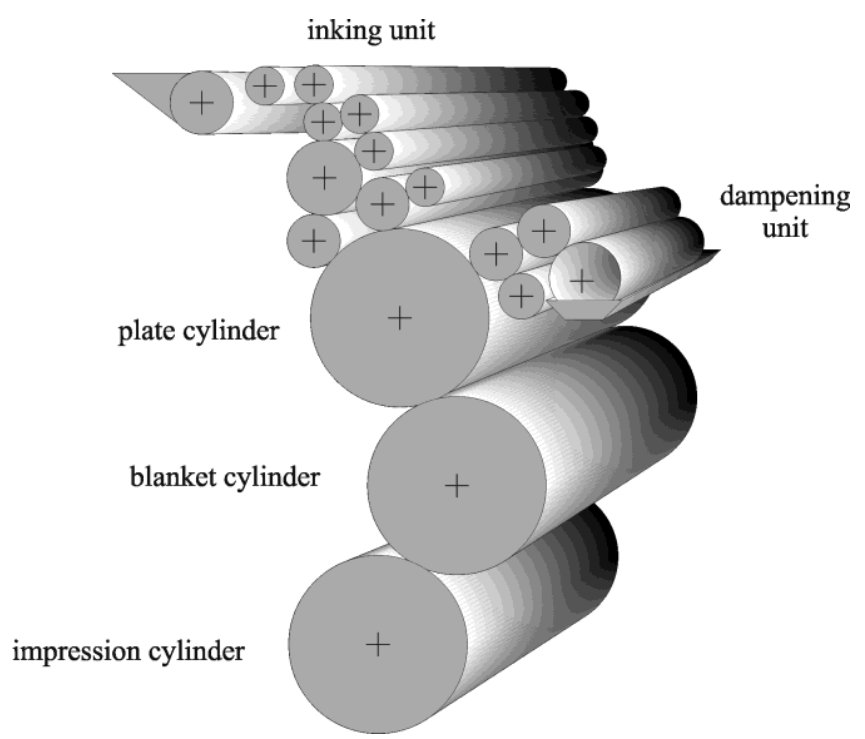

Fig. 1. An exemplary printing unit, ink and dampening systems (Jurkiewicz and Pyryev, 2011)

Many books say that it is import_ant to correctly set the pressure (Dejidas and Desree, 2005; Destree, 2005; Kipphan, 2001; Panak et al., 2002). According to those books, the pressure is meaningful for the quality of printouts and the wear and tear of consumables and machine's elements. Slurring (caused by slippage of cylinders surface), smudges on the printouts and 
the tone value increase allow for identifying the quality of parameters. This research is devoted, among others, to measure the tone value increase. Optical density is another quality parameter which depends on pressure.

We have found no articles which would describe any results of research of the printouts' quality (the optical density and the tone value increase) depending on pressure. There are similar research cases, e.g. research concerning ink squeezed outside the printing dots (Megat et al., 1997), where three values of pressure and the corresponding tone value increase are given. However, no method is specified of measuring pressure and tone value increase. Neither the article (Singh et al., 1996) specifies a method of changing pressure, so it is impossible to assess if the applied method was correct, namely if met the prerequisite requiring identical printing conditions for different pressures. The empirical equation for transfer of ink between rollers is available in Walker and Fetsko (1955).

\section{METHODS OF RESEARCH}

So far, the research problem regarding the influence of pressure on the quality of printouts was to obtain effects of printing with different pressure at identical printing conditions, such as e.g. the ink and the dampening solution balance change during printing as well as with every stop of the machine. The research described in this article fulfills the requirement of identical printing conditions. This effect was obtained in result of fixing from 0 to 5 underlay sheets to the blanket cylinder in the same moment. With this method it was possible to obtain the printing effect for one printout at 6 different pressures. A single underlay sheet was 0.28 $\mathrm{mm}$ thick.

In order to preclude the variable thickness of glue from affecting the measurement results the underlay sheets were glued to each other at one edge only, and not on the entire surface. The optimum pressure was set for 0 underlay sheets. Adequate settings of the printing machine were adjusted to the measurements taken on the printout part which corresponded to 0 underlay sheet setting.

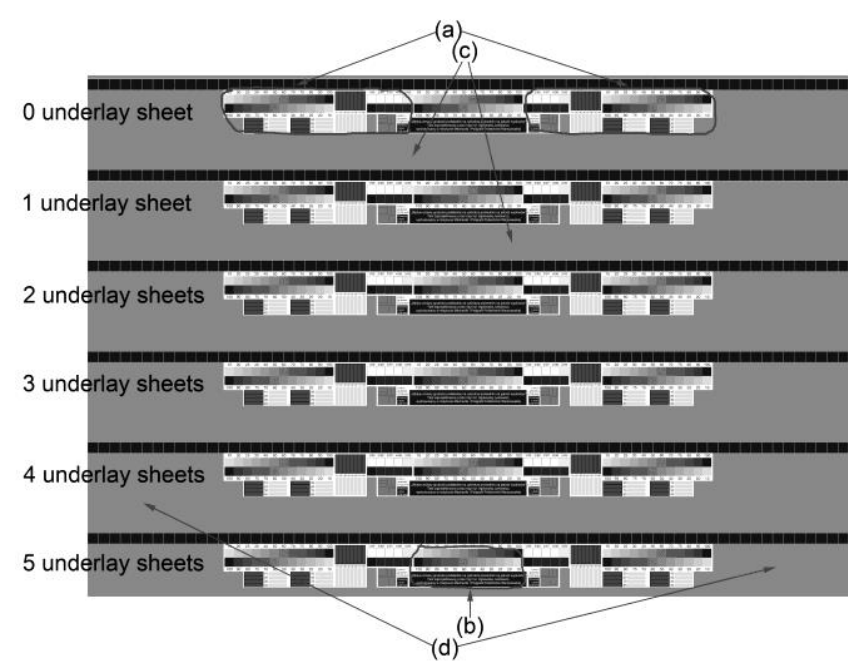

Fig. 2. Test designed for research the change of printouts' quality depending on pressure of a blanket cylinder against an impression cylinder and a plate cylinder
For purposes of this research a test has been designed in the A3 format (Fig. 2). The test consists of 6 identical rows, and each of them includes the same segment (element (a), Fig. 2) from the operator's side and from the drive's site. This segment includes two scales from $10 \%$ to $100 \%$ stepping regularly by $10 \%$ oriented in opposite directions (element (2) on the Fig. 3), the tone field values running from $1 \%$ to $5 \%$ and the fields with tone values from $95 \%$ to $99 \%$ stepping regularly by $1 \%$ (element (3), Fig. 3 ), as well as vertical and horizontal and positive and negative microlines (element (4), Fig. 3), as well as fields allowing for doubling control (element (5), Fig. 3). Next to the tone value scale stepping regularly by $10 \%$ there are additional fields with tone values of $25 \%$ and $75 \%$.

Furthermore, in the middle of printouts there is another scale stepping by $10 \%$ and a caption informing the test designer (element (b), Fig. 3). Throughout the printout width there is a control stripe with full-tone fields (element (1), Fig. 3).

The gray background between the test elements has a tone value totalling $50 \% \mathrm{~K}$ (it means $50 \%$ of black colour so it is a gray colour). It more or less corresponds to the average tone value on the test, looking along the printing. It was placed on the test to prevent from accumulating too much ink on the cylinders which is not transferred farther. Otherwise, unexpected errors might arise disturbing the research results. Between particular rows a $2 \mathrm{~cm}$ space was left (element (c) on the Fig. 2) which was covered by $50 \%$ of black colour. It was because this place is a boundary between different pressures (in the middle part of that space was a beginning of a new underlay sheet). Additionally, both sides of the test were provided with a $7 \mathrm{~cm}$ wide space (element (d), Fig. 3). Width of the underlay sheets was smaller than that of the test. This $7 \mathrm{~cm}$ wide space aims at checking if there are any unforeseen phenomena across the printing which may affect the research results.

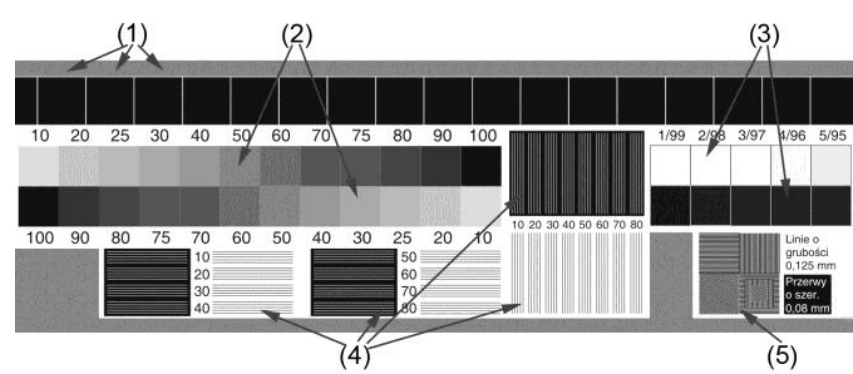

Fig. 3. Part of the test: (1) solid fields with $100 \%$ of the tone value, (2) the tone value scales from $10 \%$ to $100 \%$ stepping regularly by $10 \%$, (3) the tone value scales from $1 \%$ to $5 \%$ and from $95 \%$ to $99 \%$ stepping regularly by $1 \%$, (4) vertical and horizontal positive and negative microlines, (5) fields for controlling doubling

The plate was made using the CtP (Computer to Plate) method. It was exposed to light with a screen ruling $150 \mathrm{Ipi}$ and resolution 2400 dpi.

The designed test was printed using a sheet-fed offset printing machine Adast Dominant 515 in standardized conditions. The cyan ink was used. The printouts were made with the ink amount being changed due to reducing the ink fountain roller rotation. The printing speed was 50 printouts per minute. BT 3200 blanket by Böttcher was used which is made of 4 fabrics and a compressible layer with closed micropores. This blanket belongs to a group of blankets with rather low roughness of 0.8-1.1 $\mu \mathrm{m}$, small hardness of $78^{\circ}$ Shore, but with quite significant surface 
microhardness of $63^{\circ}$ Shore at the same time. It was very important for us to have a blanket allowing for good projection of small dots.

The described test was printed on the double coated paper with grammage of $115 \mathrm{~g} / \mathrm{m}^{2}$ and on the uncoated paper with grammage of $120 \mathrm{~g} / \mathrm{m}^{2}$.

Spectrophotometer Gretag SpectroEye was used to measure optical density using the tone value scale showing fields from $10 \%$ to $100 \%$ with a single step by $10 \%$. The optical density for nontransparent materials is a common logarithm of ratio between the amount of light falling on the sample and the amount of light reflected (Sharma and Pietrzak, 2006).

For each field 3 measurements were taken to reduce a risk of measurement errors. The spectrophotometer was set as follows: Illuminant $\mathrm{A}$, Observer $2^{\circ}$, density standard DIN, polarizing filter and as a standard whiteness - paper. The measurements were taken in 3 parts of the printout: from the drive's side, in the middle part and from the operator's side.

For fields of the tone value scale from $10 \%$ to $100 \%$ stepping regularly by $10 \%$ the tone value S [\%] was computed with the Murray-Davies equation (ISO Standard 12647-1, 2004):

$S=100 \cdot\left(\frac{1-10^{-\left(D_{t}-D_{0}\right)}}{1-10^{-\left(D_{S}-D_{0}\right)}}\right)$

where: $D_{0}$ - optical density of the unprinted print substrate (paper), $D_{t}$ - optical density of the half-tone, $D_{S}$ - optical density of the full-tone.

Because in the spectrophotometer the paper was selected as a standard whiteness, the device automatically showed $\left(D_{t}-D_{0}\right)$ and $\left(D_{S}-D_{0}\right)$.

Several hundred printouts for each type of paper were made. For measurement purposes over a dozen printouts were selected. The criterion of selection was the optical density of the middle field of the control stripe including full-tone fields placed in the row for 0 underlay sheets. Several printouts were selected with too big amount of ink, too small amount of ink, and more printouts featured with nearly correct optical density.

According to recommendations of Heidelberg we assumed for the uncoated paper that a correct printout was the one whose fulltone field's optical density totalled 1.05 . The following printouts were selected: 1.40 (1.392), 1.30 (1.311), 1.18 (1.189), 1.10 (1.107), 1.09 (1.085), 1.08 (1.073), 1.07 (1.066), 1.06 (1,061), 1.05 (1.055), 1.04 (1.050), 1.03 (1.049), $1.02(1.022), 1.01$ (1.014), 1.00 (1.002), 0.09 (0.904), 0.08 (0.814), 0.07(0.713). The brackets show the average optical density of full-tone fields of the control stripe which is placed in this row for 0 underlay sheets.

According to recommendations of Heidelberg we assumed for the coated paper that a correct printout was the one whose fulltone field's optical density totalled 1.55 . The following printouts were selected: 1.88 (1.806), 1.81 (1.753), 1.71 (1.714), 1.61 (1.562), 1.59 (1.539), 1.58 (1.549), 1.57 (1.542), 1.56 (1.532), 1.55 (1.513), 1.54 (1.526), 1.53 (1.539), 1.52 (1.509), 1.51 (1.521), 1.47 (1.475), 1.40 (1.443), 1.30 (1.325), 1.20 (1.154), 1.10 (1.058).

For purposes of this analysis the measurements of the optical density and of the tone value increase taken for three tone value scales in one row were averaged. This allowed for eliminating the measurement errors. The tone value increase is a difference between the tone value on the original copy and the tone value on the printout (ISO Standard 12647-1, 2004), thus meaning enlargement of printing dots on paper versus those visible on the original copy.

\section{RESULTS OF RESEARCH}

In this article results of measered the optical density and computed the tone value increase from this optical density are performed. For both kind of papers graphs for the optical density for field 100\% (Fig. 4, Fig. 5, Fig. 10, Fig. 11), for field 50\% (Fig. 6 , Fig. 7, Fig. 12, Fig. 13) and for the tone value increase for field $50 \%$ Fig. 8, Fig. 9, Fig. 14, Fig. 15) are shown. These graps are divided on two groups: for different amount of ink (Fig. 4, Fig. 6, Fig. 8, Fig. 10, Fig. 12, Fig. 14) and for amount of ink near optimal (Fig. 5, Fig. 7, Fig. 8, Fig. 9, Fig. 10, Fig. 11).

\subsection{Uncoated paper}

Graph illustrating the optical density for field 100\% for printouts with different amount of ink (Fig. 4) shows that the optical density hardly varies with changing pressure. A slight increase of the optical density can be seen for printouts only with a very big amount of ink (for a printout with the optical density of the middle field equal 1.40) and with a very small amount of ink (for a printout with the optical density of the middle field equal $0.70)$.

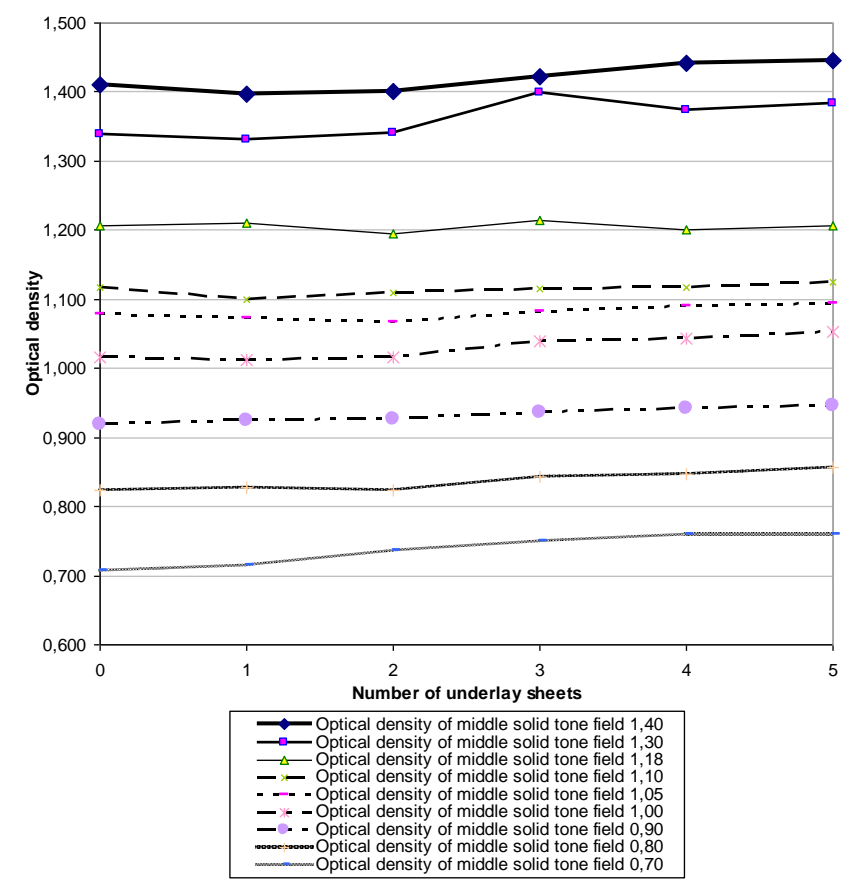

Fig. 4. The optical density for field $100 \%$ for different amount of ink for uncoated paper depending on the number of underlay sheets

On the graph for a printout with a correct amount of ink (Fig. 5) can be seen that the optical density shows some insignificant fluctuations. This is caused by unevenness of cylinders or of the blanket (unevenness of blanket allowable by the manufacturer is $+/-0.02 \mathrm{~mm}$ ).

The graph illustrating the optical density for field $50 \%$ with respect to printouts with different amount of ink (Fig. 6) shows that a difference in the optical density is bigger than that shown by the corresponding graph for field $50 \%$ (Fig. 4). This is true in case of too much amount of ink and for near-to-correct amount of ink. This most likely results from that the printing dots for field $50 \%$ are 
smaller than those for field $100 \%$ which makes the ink easier to spill outside the printing dots on the blanket or on the paper.

The difference between the optical density for field $50 \%$ with respect to printouts with correct amount of ink (Fig. 7), for the biggest and the smallest pressure, is bigger than that for field $100 \%$. But for underlay sheets from 0 to 4 this function fluctuates and shows similar values in both minima and maxima. Only for 5 underlay sheets the optical density visibly increases. This means that only with changing underlay sheets by about $0.1 \mathrm{~mm}$ (exactly $0.112 \mathrm{~mm}$ ) is of significance. Most curves show that, despite fluctuations, the function usually increases for 2 underlay sheets.

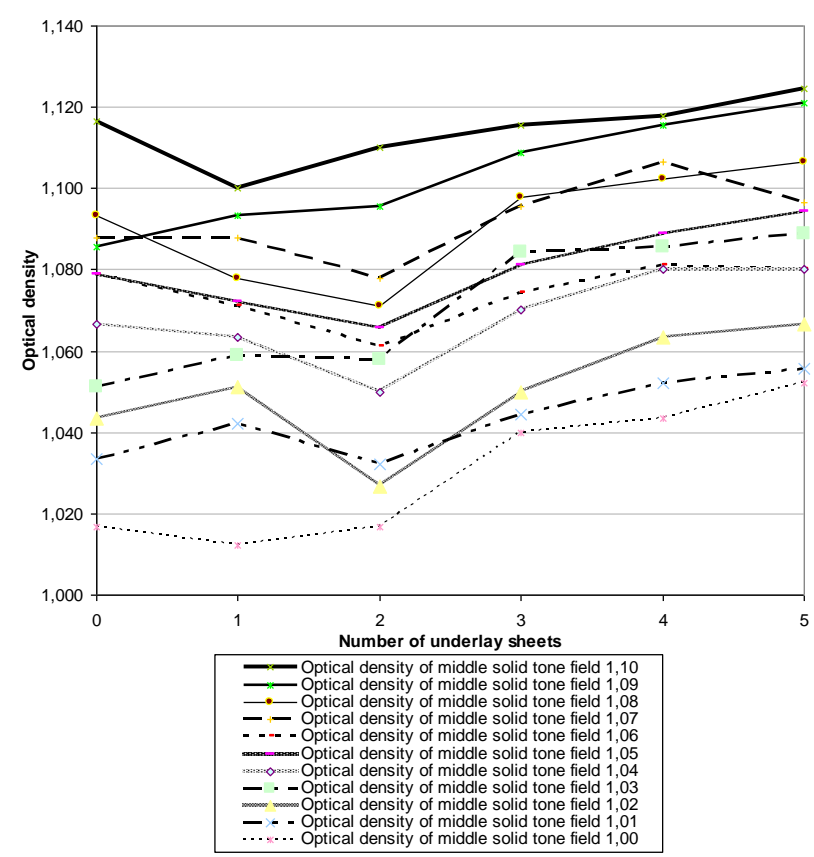

Fig. 5. The optical density for field $100 \%$ for correct amount of ink for uncoated paper depending on the number of underlay sheets

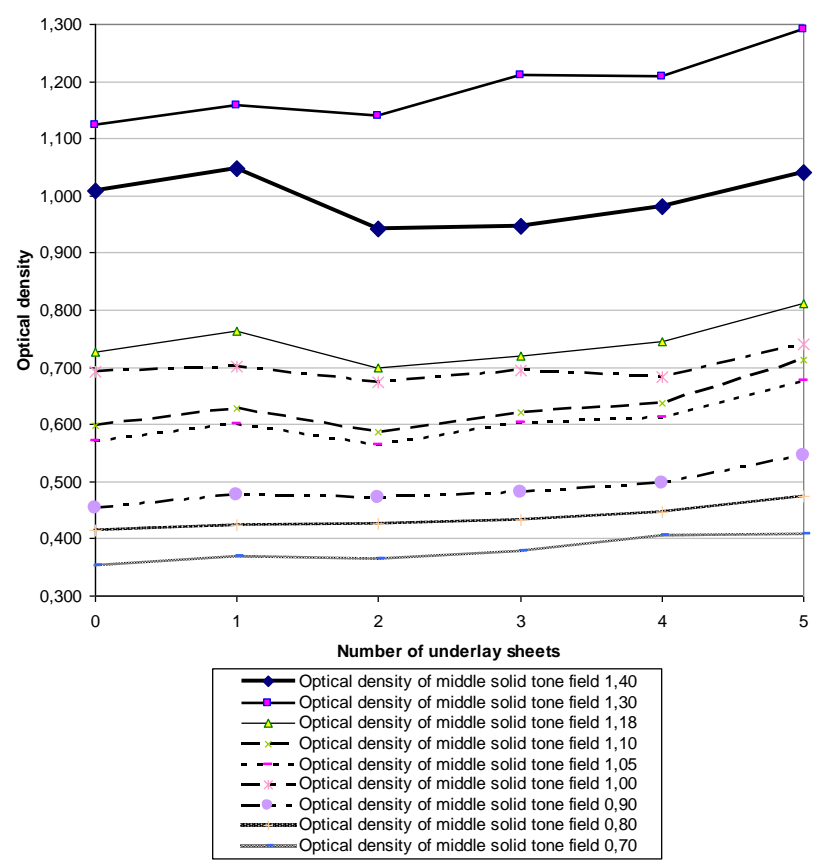

Fig. 6. The optical density for field $50 \%$ for different amount of ink for uncoated paper depending on the number of underlay sheets
Curves for the tone value increase for field $50 \%$ for different amount of ink (Fig. 8) show that usually the minimum is for 2 underlay sheets.

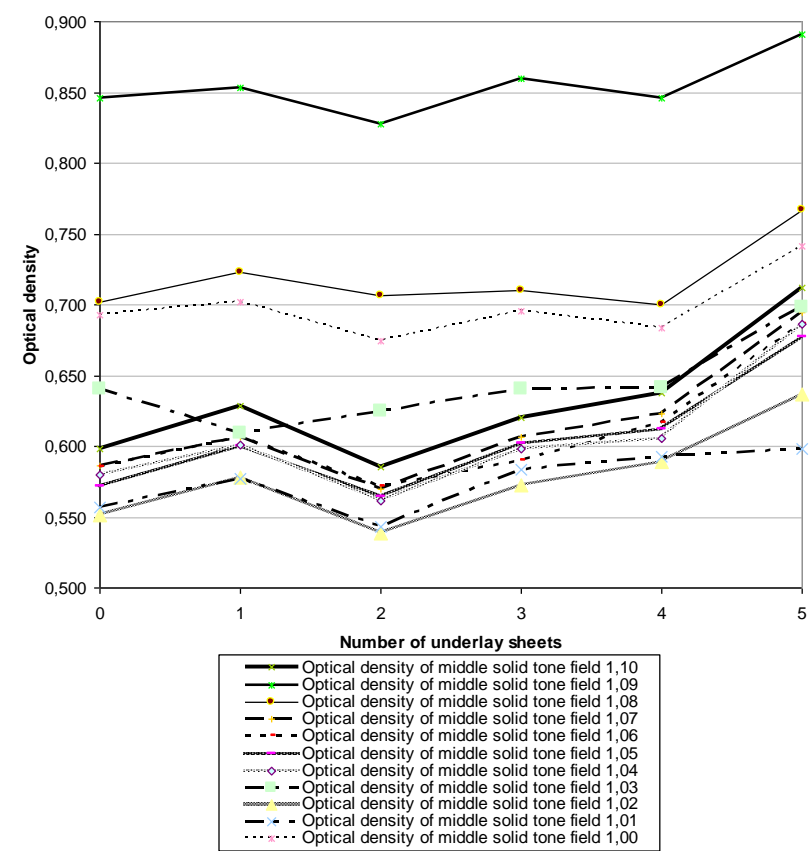

Fig. 7. The optical density for field $50 \%$ for correct amount of ink for uncoated paper depending on the number of underlay sheets

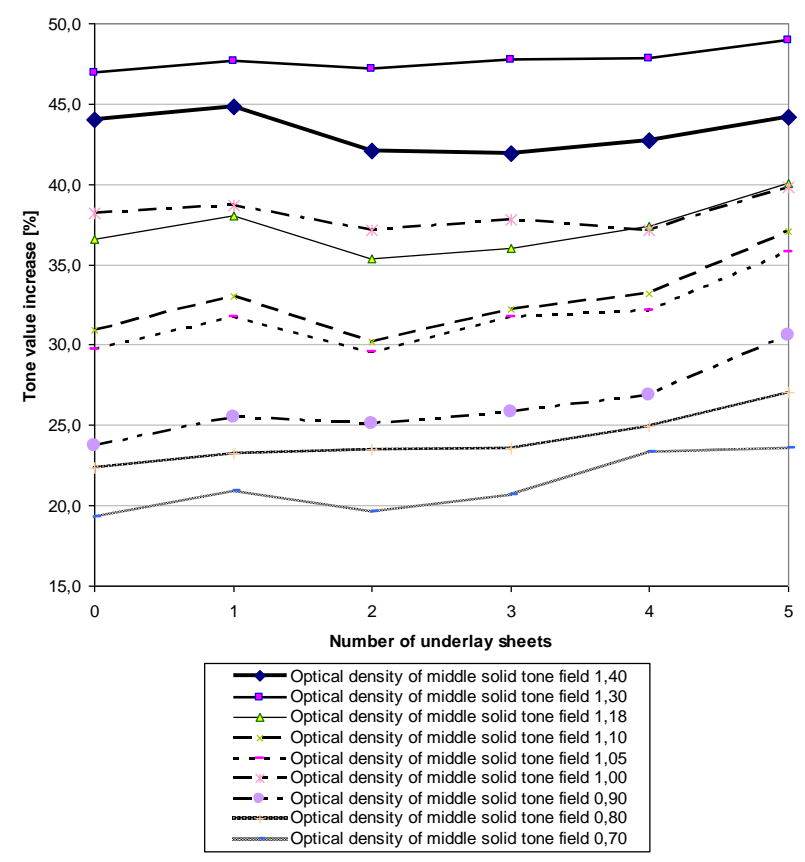

Fig. 8. The tone value increase for field $50 \%$ for different amount of ink for uncoated paper depending on the number of underlay sheets

It is interesting that all curves show the maximum for 1 underlay sheet (Fig. 8). As this takes place repeatedly for each curve it is most likely caused by uneven cylinder or uneven blanket in that particular place (identically as for the optical density for field $50 \%$ ). Measurement of pressure between the plate cylinder and the blanket cylinder (Tab. 1) confirms this way of reasoning. Results for 1 underlay sheet stand out clearly. There is a significant difference between value of measurement taken 
from the operator's side, on the central part of cylinder, and that from the drive's side. Moreover, the pressure on the central part of cylinder and from the operator side is bigger than that for 0 underlay sheets. For measurement purpose the Pressure Indicator was used. In order to ensure reliable measurement values the underlay sheet with thickness identical to that of the indicator was removed during measuring the pressure.

Tab. 1. The measurements of pressure between the plate cylinder and the blanket cylinder for each number of underlay sheets

\begin{tabular}{|c|c|c|c|}
\hline $\begin{array}{c}\text { Number of } \\
\text { underlay } \\
\text { sheets }\end{array}$ & $\begin{array}{c}\text { Measurement } \\
\text { from the } \\
\text { operator's } \\
{\left[\mathbf{N} / \mathbf{c m}^{2}\right]}\end{array}$ & $\begin{array}{c}\text { Measurement } \\
\text { on the centre } \\
{\left[\mathbf{N} / \mathbf{c m}^{2}\right]}\end{array}$ & $\begin{array}{c}\text { Measurement } \\
\text { from the } \\
\text { drive's site } \\
{\left[\mathbf{N} / \mathbf{c m}^{2}\right]}\end{array}$ \\
\hline 0 & 480 & 340 & 360 \\
\hline 1 & 590 & 330 & 250 \\
\hline 2 & 560 & 470 & 430 \\
\hline 3 & 550 & 490 & 460 \\
\hline 4 & 620 & 570 & 550 \\
\hline 5 & 660 & 610 & 600 \\
\hline
\end{tabular}

The run of most curves illustrating the tone value increase for field $50 \%$ for correct amount of ink (Fig. 9) is identical to that of curves for different amount of ink. A maximum difference of the tone value increase between the lowest and the biggest point on the curves is $7 \%$.

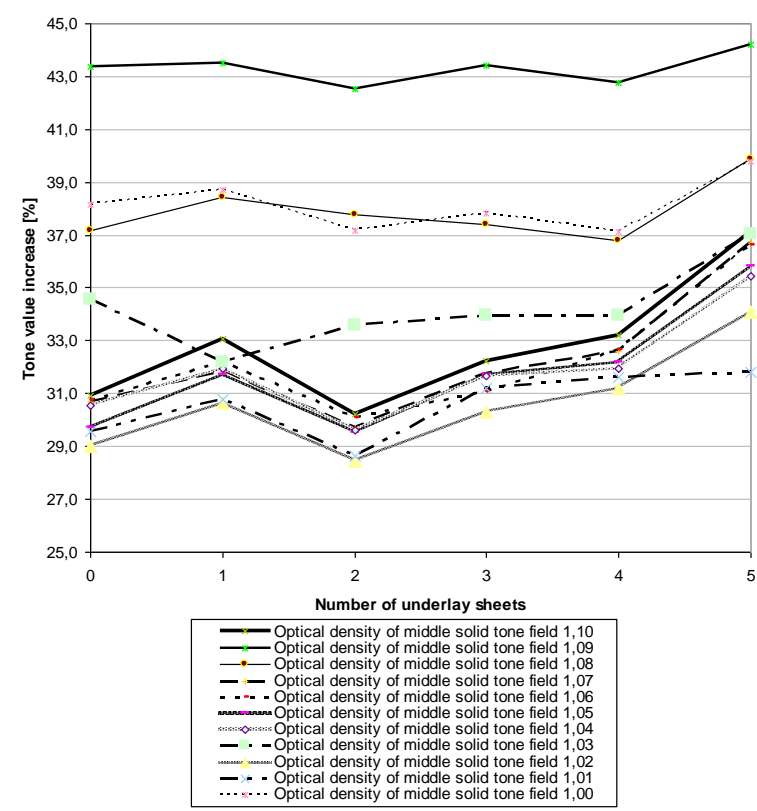

Fig. 9. The tone value increase for field $50 \%$ for correct amount of ink for uncoated paper depending on the number of underlay sheets

A maximum difference between the tone value increase for 0 underlay sheet and for 5 underlay sheets is 7\% (Fig 9). According to Table 5 in 12647-2 ISO Standard (ISO Standard 12647-2, 2004) for screen ruling $60 \mathrm{l} / \mathrm{cm}$ the deviation in the tone value increase between printouts during production can be maximum $5 \%$. If we take this value as a limit for changing of the tone value increase, the value $7 \%$ for uncoated paper is not negligible. These values are for printouts printed with correct amount of the ink. Graphs illustrating the tone value increase for uncoated paper (Fig. 9) show that when the clamp is increased by 4 underlay sheets $\left(4^{*} 0.028 \mathrm{~mm}=0.112 \mathrm{~mm}\right)$, the difference in the tone value increase is much lower. It is, for the correct amount of ink, from $0 \%$ to $2 \%$. It means that when the thickness of underlay sheets will be increased by about $0.1 \mathrm{~mm}$, the quality of printouts do not change significantly.

\subsection{Coated paper}

Comparing graphs illustrating optical density for different amount of ink for field $100 \%$ for both types of paper we can see that the difference in the optical density for the smallest and the biggest pressure is bigger for coated paper (Fig. 10). Starting from printouts with the optical density of the middle field equals 1.61 the minimum of function is for 1 underlay sheet and moves to the right for up to 3 underlay sheets with respect to printouts with the optical density of the middle field equal 1.10. In the case of too much amount of ink for a printout with the optical density of the middle field equal 1.81 the minimum is for 1 underlay sheet, and for a printout with the optical density of the middle field equal 1.71 the minimum is for 2 underlay sheets. Only the curve for the biggest amount of ink shows the run which significantly differs from the other ones. This curve seems to show that starting from 2 underlay sheets the amount of the transferred ink decreases.

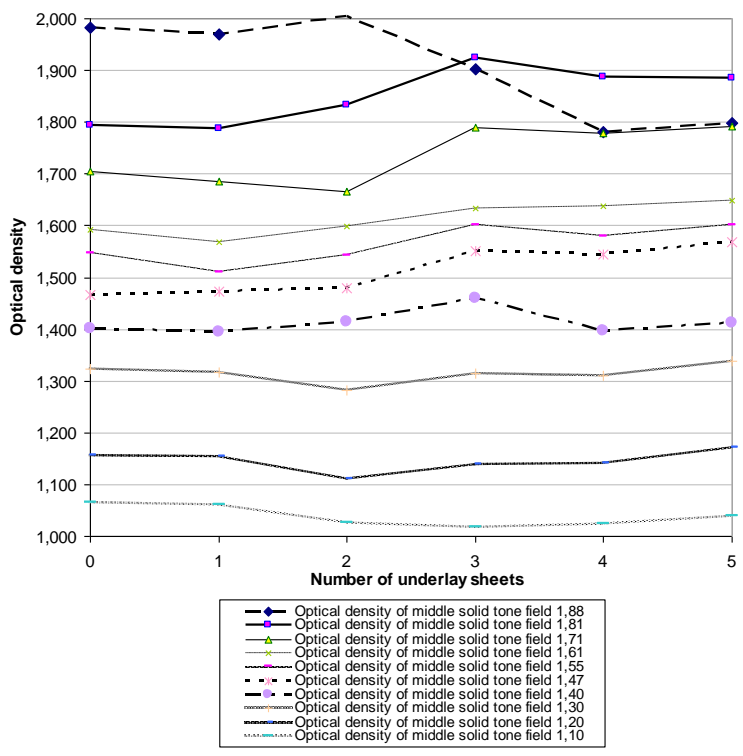

Fig. 10. The optical density for field $100 \%$ for different amount of ink for coated paper depending on the number of underlay sheets

As regards curves illustrating the optical density for field 100\% for correct amount of ink (Fig. 11) the difference between maximum and minimum is very slight, namely it is smaller than 0.1 . These fluctuations are most likely result from unevenness of cylinders or of blanket.

Likewise in the case of uncoated paper the difference between the highest and the lowest point on the curve illustrating the optical density for field $50 \%$ (Fig. 12) is bigger than for field $100 \%$. With respect to the coated paper the optical density for field $50 \%$ depends more on pressure than with respect to the uncoated paper. This optical density increases when the number of underlay sheets increases. The exception is observed with respect to 1 underlay sheet, but this place on graphs for uncoated paper and for measurements of pressure stands out too. This confirms 
the hypothesis that in a place with 1 underlay sheet the cylinder or the blanket was uneven. Only one curve differs from the others, namely the one for the biggest amount of ink. For that curve the fluctuations are so big that it is difficult to find one trend merely.

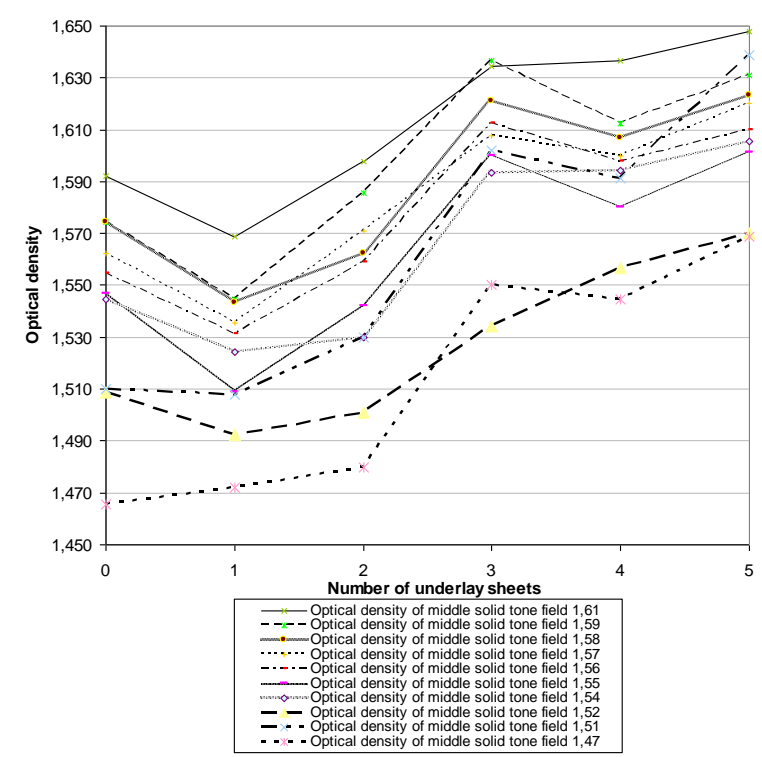

Fig. 11. The optical density for field $100 \%$ for correct amount of ink for coated paper depending on the number of underlay sheets

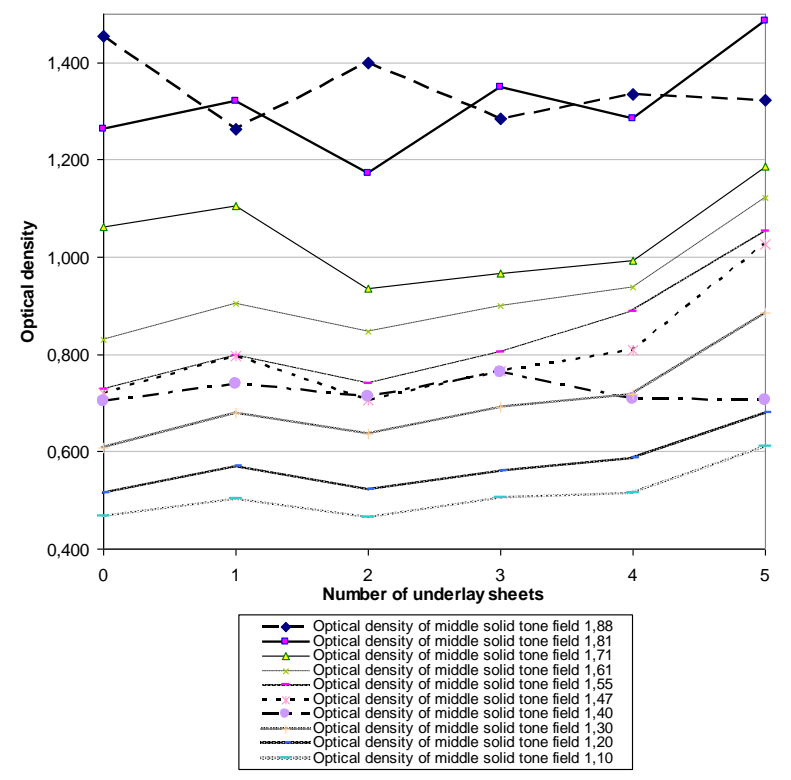

Fig. 12. The optical density for field $50 \%$ for different amount of ink for coated paper depending on the number of underlay sheets

Apparently, for from 2 up to 5 underlay sheets the curves illustrating the optical density for correct amount of ink (Fig. 13) increase by $0.25-0.35$.

Most of the curves on the graph illustrating the tone value increase for field $50 \%$ for different amounts of ink (Fig. 14) show local minimum for 1 underlay sheet, and for more than 2 underlay sheets these functions increase. The run of most of those curves is similar to that of most curves on the corresponding graph for uncoated paper. But as regards graphs for coated paper the difference between the biggest and the lowest value is bigger by about $5 \%$ than that for uncoated paper.
The run of curves for the tone value increase for field $50 \%$ (Fig. 15) for correct amount of ink is similar to that of curves for the optical density for field $50 \%$ (Fig. 9). The difference between minimum and maximum on these curves oscillates from $8 \%$ to $10 \%$. These values are not negligible, if we take $5 \%$ (the deviation in the tone value increase between printouts during production according to Table 5 in (12647-2 ISO Standard)) as a criterion. It is more than for uncoated paper. When the clamp is increased by 4 underlay sheets $\left(4^{*} 0.028 \mathrm{~mm}=0.112 \mathrm{~mm}\right)$, the difference in the tone value increase is much lower. It is, for the correct amount of ink, from $3 \%$ to $5 \%$. It means that when the thickness of underlay sheets will be increased by about $0.1 \mathrm{~mm}$, the quality of printouts does not change significantly - the same like for uncoated paper.

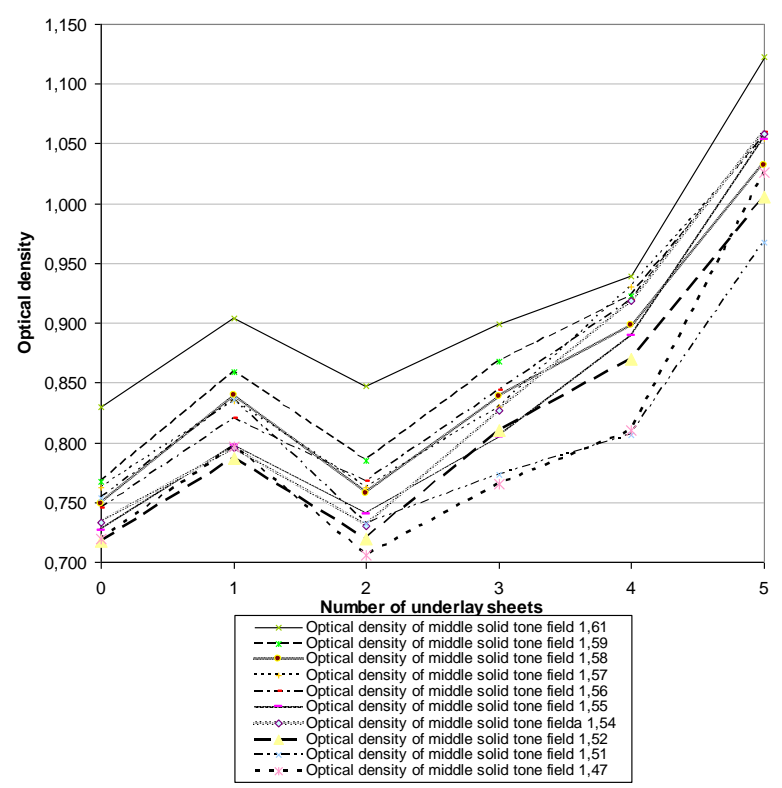

Fig. 13. The optical density for field $50 \%$ for correct amount of ink for coated paper depending on the number of underlay sheets

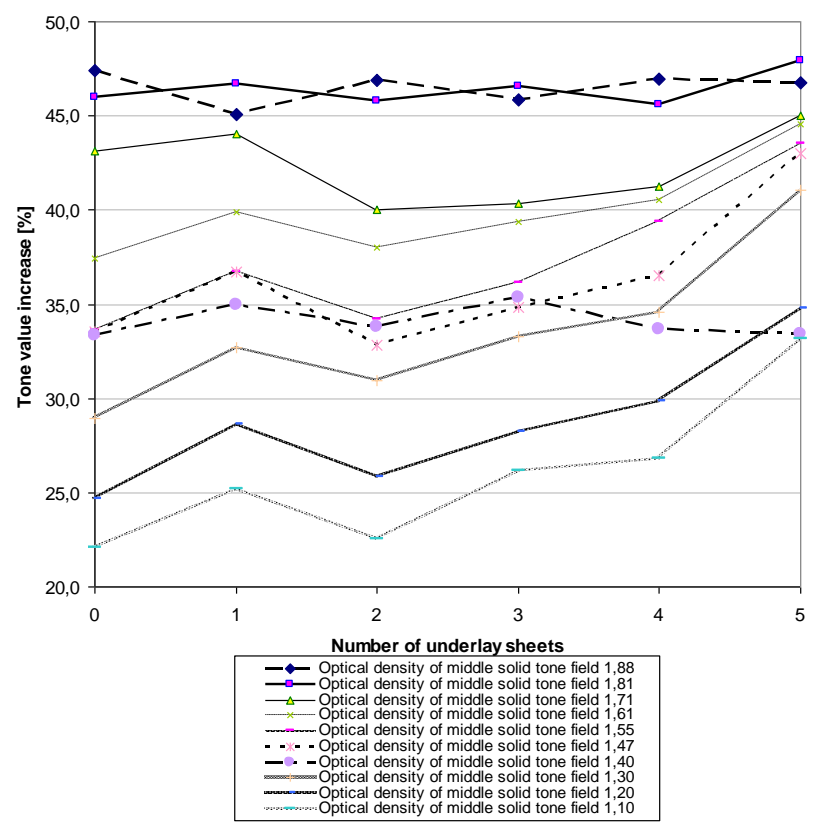

Fig. 14. The tone value increase for field $50 \%$ for different amount of ink for coated paper depending on the number of underlay sheets 


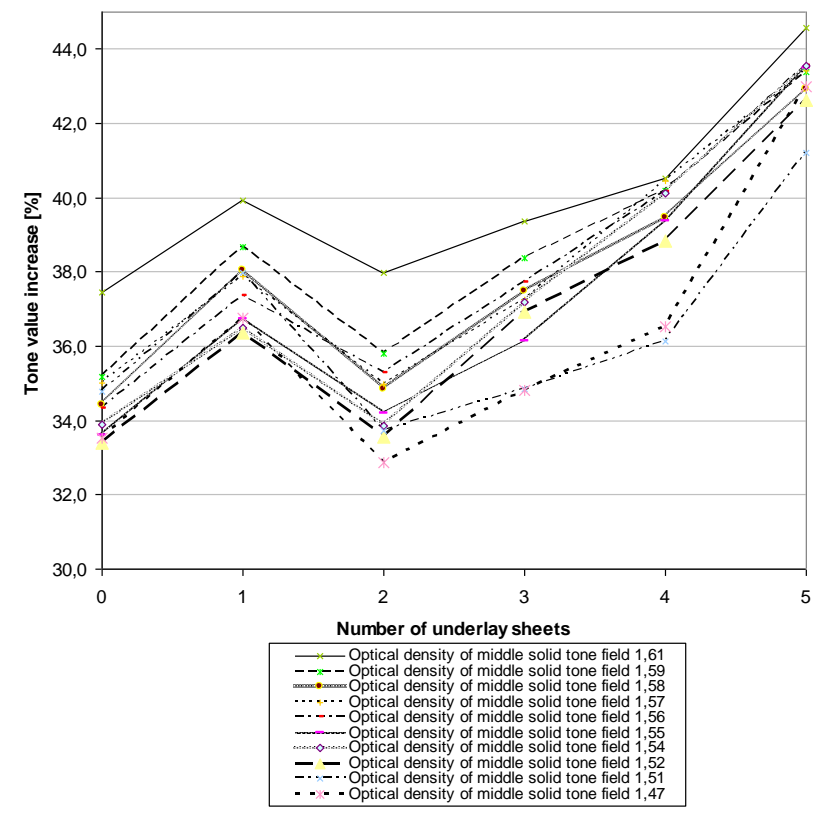

Fig. 15. The tone value increase for field $50 \%$ for correct amount of ink for coated paper depending on the number of underlay sheets

\section{CONCLUSION}

For both types of paper the optical density and the tone value increase slightly changes above the optimal pressure recommended by the manufacturer. It means that the engineering solution, which is the compressible blanket, is very good.

For both types of paper the difference of the optical density value depending on the number of underlay sheets is bigger for field $50 \%$ than for field $100 \%$. This results from that ink has more place to spill on field $50 \%$ than on field $100 \%$ where dots occupy the entire surface of that field, whereas they do not occupy the entire surface of field $50 \%$ (the dots occupy half of the surface of the original copy, and more than half of the surface of the blanket or of the paper). The difference in the value of optical density for field $100 \%$ is, depending on pressure, not bigger than 0.1 , except for where the amount of ink for coated paper is big thus making the difference bigger. Therefore, we can assume that the said difference in value of the optical density is referred to as an incidental fluctuation for most of curves and we can conclude that the optical density of fields $100 \%$ does not depend on pressure above its optimal value.

Only for 5 underlay sheets the optical density and the tone value increase visibly goes up for field $50 \%$. This means that only where the underlay sheet thickness equals about $0.1 \mathrm{~mm}$ it may affect the values of the optical density and the tone value increase.

With respect to the coated paper the differences for the optical density and for the tone value increase are, depending on pressure, bigger than with respect to the uncoated paper. This result indicates that the increasing pressure makes ink rather spill out side the printing dots, thus enlarging the dots than being pressed into paper. This is also confirmed by the curves, very similar in their run, illustrating the optical density and the tone value increase for field $50 \%$. Therefore, changes in the value of the optical density correspond to those in size of dots.

The used method of obtaining different pressure on one printout was found to work, because it was possible to come to different printing results with different pressure under identical printing conditions. We obtain different pressure and the same printing condition for the one printout.

The above involves a necessity to make further research to find out if e.g. in a place with 1 underlay sheet the so big change in the optical density value was caused by that 1 underlay sheet or rather by unevenness of cylinders or of the blanket (as indicated by the pressure measurement). It is planned to examine sections of the printouts under a microscope to see how ink soaks in and spills on the paper.

\section{REFERENCES}

1. Dejidas L. P., Desree T. M. (2005), Sheetfed Offset Press Operating, Graphic Arts Technical Fndtn, Portland.

2. Destree T. M. (2005), The Pia/Gatf Guide to Troubleshooting for the Sheetfed Offset Press, Gatfpress.

3. ISO Standard 12647-1 (2004), Graphic technology - Process control for the production of half-tone colour separations, proof and production prints - Part 1: Parameters and measurement methods, Switzerland.

4. ISO Standard 12647-2 (2004), Graphic technology - Process control for the production of half-tone colour separations, proof and production prints - Part 2: Offset processes, Switzerland.

5. Jurkiewicz A., Pyr'yev Y. (2011), Analysis of stress problem between two rollers or two cylinders in sheet-fed offset printing machine, Acta Mechanica et Automatica, Vol. 5, No 4., 58-61.

6. Kipphan H. (2001), Handbook of Print Media, Springer-Verlag, Berlin Heidelberg.

7. Megat Ahmedy M. M. H. et al. (1997), A model for ink impression into a porous substrate, J. Phys. D: Appl. Phys., 30, 2276-2284.

8. Panak J. et al. (2002), Printing processes and technology, COBRPP, Warsaw (in Polish).

9. Sharma A., Pietrzak A. (2006), Understanding color management, Ergo BTL, Warsaw (in Polish).

10. Singh S. P. et al. (1996), Surface characterization by partialcoverage printing and image analysis, Process \& Product Quality Conference, 119-127.

11. Walker W. C. and Fetsko J. M. (1955), A concept of Ink transfer during printing, American Ink Maker, 33, 69-71.

This work has been supported by the European Union in the framework of the European Social Fund through the Warsaw University of Technology Development Programme by the scholarship awarded by the Center of Advanced Studies for Agnieszka Jurkiewicz.

Project partially supported by the National Science Centre, grant No. DEC-2011/03/B/ST8/06478. 